\title{
Encapsulated Follicular Variant of Papillary Thyroid Carcinoma with Bone Metastases
}

\author{
Zubair W. Baloch, M.D., Ph.D., Virginia A. LiVolsi, M.D. \\ Department of Pathology \& Laboratory Medicine, University of Pennsylvania Medical Center, \\ Philadelphia, Pennsylvania
}

\begin{abstract}
Although true follicular thyroid carcinoma is known to metastasize via the bloodstream and give rise to bone and lung metastases, such a pattern of spread is rare in papillary thyroid carcinoma. The follicular variant of papillary thyroid carcinoma (FVPTC) is believed to behave in a clinical manner similar to usual or classical papillary cancer and to follow a similar indolent course.

There have been a few reports of "aggressive" FVPTC wherein follicular patterned tumors with nuclear features of papillary carcinoma have metastasized hematogenously; these neoplasms have been diffusely invasive or multicentric in the thyroid.

We report five cases of FVPTC, which were encapsulated and simulated grossly and microscopically follicular adenomas. In two of these, the primary was discovered after clinical presentation of bone metastases. In three others, bony metastases (without other nonosseous metastases) arose 7 to 17 years after thyroid lobectomy for lesions initially diagnosed as follicular adenoma. In retrospect, these three encapsulated lesions had vascular invasion. We wish to bring attention to these innocuousappearing lesions, which, although sharing nuclear features of papillary cancer, behave clinically in an unexpectedly malignant fashion.
\end{abstract}

KEY WORDS: Bone metastasis, Follicular variant, Papillary carcinoma, Thyroid.

Mod Pathol 2000;13(8):861-865

The differential diagnosis of encapsulated follicular patterned lesions of the thyroid includes follicular adenoma, follicular carcinoma, and follicular variant of papillary thyroid carcinoma (FVPTC). All of

Copyright (C) 2000 by The United States and Canadian Academy of Pathology, Inc.

VOL. 13, NO. 8, P. 861, 2000 Printed in the U.S.A.

Date of acceptance: March 1, 2000.

Address reprint requests to: Zubair W. Baloch, MD., Ph.D., Assistant Professor, Department of Pathology and Laboratory Medicine, 6 Founders Pavilion, 3400 Spruce Street, Philadelphia, PA 19104; e-mail: baloch@ mail.med.upenn.edu; fax: 215-349-8994. these lesions exhibit similar clinical presentations and gross morphology (1-4). The diagnosis of follicular carcinoma requires the presence of capsular and/or vascular invasion (1-5) and that of FVPTC depends on the finding of appropriate nuclear features of papillary thyroid carcinoma (PTC) (1-12).

Lindsay (10) first coined the term FVPTC, and later Chen and Rosai (11) reported the detail morphologic description of this tumor. It has been shown that a majority of these lesions behave similarly to classic PTC $(1,2,8,9)$. Morphologically, these tumors can appear partially or completely encapsulated $(1-3,5,8,9,11,12)$. The encapsulated type of FVPTC can be mistaken for follicular adenoma if totally confined within the tumor capsule or follicular carcinoma when associated with capsular and/or vascular invasion $(1-3,5,7)$. In our experience, this misdiagnosis mostly is made in a subset of encapsulated FVPTC, which usually show multifocal rather than diffuse distribution of nuclear features of PTC $(2,7,12)$.

We present five cases of encapsulated type of FVPTC that showed distant bone metastasis; three initially were diagnosed as follicular adenoma and in two the tumors initially presented with bone metastases. Three lesions showed focal vascular invasion.

\section{MATERIALS AND METHODS}

All cases were from the personal consultation files of one of the authors (VAL). Hematoxylin and eosin-stained sections were available for review in each case, and immunostains for thyroglobulin were reviewed in four cases.

The information extracted from the patient records and consultation correspondence included sex, age, clinical presentation, size of tumor, location of tumor, previous diagnoses, extent of surgery (partial versus total thyroidectomy), vascular invasion, lymph node status, and size and location of metastasis. 


\section{RESULTS}

Clinical findings and main pathologic features are described in Table 1. All patients were women, ranging in age from 40 to 79 years (mean, 61.2 years).

\section{Cases 1 and 2}

Two patients (ages 69 and 75 years) presented initially with bone metastases; in one, there was $3.0-\mathrm{cm}$ mass in left ischium and in the other a lytic lesion in right ischium. Fine-needle aspiration was performed in both and showed tumor with microfollicular growth pattern; positive thyroglobulin stains confirmed metastasis from a thyroid primary. In each case, the patient underwent total thyroidectomy.

\section{Case 3}

A 79-year-old woman underwent total thyroidectomy in 1982 for a thyroid tumor, which was diagnosed as follicular adenoma. The patient was disease-free for 15 years until she presented with a lytic thoracic spine lesion. A core biopsy showed a tumor with solid and follicular growth pattern; stain for thyroglobulin was positive, consistent with a metastasis from thyroid primary.

\section{Case 4}

A 40-year-old woman in 1980 underwent a right lobectomy and isthmectomy for a removal of right thyroid tumor. The tumor was diagnosed as follicular adenoma. In 1997, the patient presented with diffuse bony lesions throughout her body; radionuclide studies showed that these took up radioactive iodine and were diagnosed as metastases from a thyroid primary. A bone biopsy was performed, which was consistent with the radiologic impression. A subsequent CT scan also showed multiple lung nodules. The patient was treated with radioactive iodine and external radiation.

\section{Case 5}

A 43-year-old woman in 1992 underwent left thyroid lobectomy for a thyroid nodule, which was diagnosed as microfollicular adenomatoid nodule. In 1998, the patient presented to an orthopedic surgeon for back pain. A magnetic resonance imaging study demonstrated a lytic lesion in the body of the third lumbar vertebra. This was confirmed by CT scan, which showed a lytic bony lesion with extension into the left psoas muscle and adjacent spinal canal. A fine-needle aspiration showed a tumor with follicular growth pattern, and thyroglobulin immunostain was positive. This morphology and immunoprofile was consistent with metastasis from thyroid primary.

\section{Pathologic Findings}

In four cases, the primary tumors were solitary thyroid nodules; three were in right lobe, and laterality was unknown in one case. In one case, the tumors were present in both right and left lobes and measured 0.7 and $1.5 \mathrm{~cm}$, respectively. All tumors were thickly encapsulated with varying degrees of intratumoral fibrosis (Fig. 1A, B). In the case with bilateral nodules, the tumors showed extensive fibrosis and calcification with residual tumor present at the periphery of the tumor nodule. Multifocal small foci of papillary microcarcinoma follicular variant were present in one case. In all cases, the uninvolved thyroid parenchyma showed degenerative and hyperplastic changes consistent with nodular goiter and foci of chronic nonspecific thyroiditis.

All tumors showed a follicular growth pattern consisting of follicles of varying sizes and shapes that contained thick eosinophilic luminal colloid

TABLE 1. Clinical Features of Patients with FVPTC and Bone Metastases

\begin{tabular}{|c|c|c|c|c|c|}
\hline Case & Age/Sex & Presentation & Treatment & Thyroid & $\mathrm{VI}$ \\
\hline 1 & $69 / \mathrm{F}$ & $\begin{array}{l}\text { 3.0 }-\mathrm{cm} \text { L1 solid mass } \mathrm{w} / \text { nerve compression. } \mathrm{FNA}= \\
\text { microfollicular thyroid tumor; thyrog }+\end{array}$ & Total thyr & $\begin{array}{l}2 \text { encap lesions } 0.7 \& 1.5 \mathrm{~cm} \text {; } \\
\text { multifocal PTC Mca }\end{array}$ & - \\
\hline 2 & $75 / F$ & $\begin{array}{l}\text { Lytic lesion right ischium. FNA = thyroid mets; } \\
\text { thyrog }+\end{array}$ & Total thyr & Encap lesion $1.5 \mathrm{~cm}$ & - \\
\hline 3 & $79 / F$ & $\begin{array}{l}\text { Thyroid tumor, diagnosed as follicular adenoma } \\
\text { (1982). Spine lesion 1997; thyroid mets, thyrog + }\end{array}$ & Total thyr & Encap lesion; size? & + \\
\hline 4 & $40 / \mathrm{F}$ & $\begin{array}{l}\text { Right thyroid tumor diagnosed as follicular } \\
\text { adenoma (1980). Diffuse bony mets } 1997 \\
\text { diagnosed by imaging studies and radioactive } \\
\text { iodine uptake. Bone biopsy: thyroid mets, thyrog } \\
\text { +. Subsequent CT scan: lung mets }\end{array}$ & Right thyr (1980) & Encap lesion $1.5 \mathrm{~cm}$ & + \\
\hline 5 & $43 / \mathrm{F}$ & $\begin{array}{l}\text { Left thyroid tumor diagnosed as adenomatoid } \\
\text { nodule (1992). L3 bone lesion; FNA biopsy: } \\
\text { thyroid mets, thyrog }+\end{array}$ & Left thyr (1992) & Encap lesion $1.1 \mathrm{~cm}$ & + \\
\hline
\end{tabular}

VI, vascular invasion; FNA, fine-needle aspiration; thyr, thyroidectomy; Mca, microcarcinoma; encap, encapsulated; mets, metastasis; thyrog, immunostains for thyroglobulin; PTC, papillary thyroid carcinoma. 

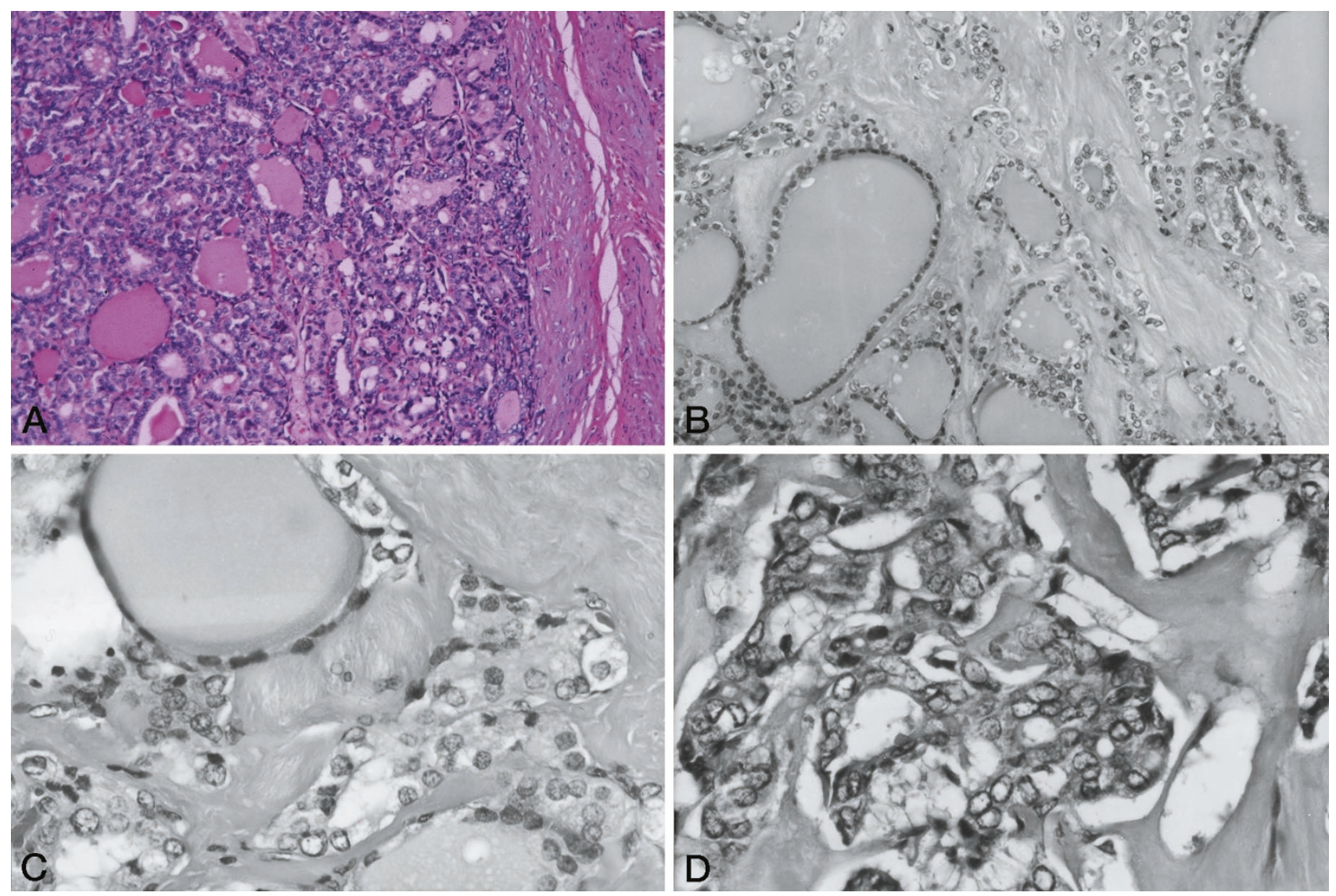

FIGURE 1. Case 3. Low-power view showing a encapsulated follicular patterned lesion (A). B, high-power view showing follicles of varying sizes and areas of sclerosis. C, D, high-power views showing follicles with and without nuclear features of papillary carcinoma.

(Fig. 1B). Multifocally throughout these nodules, the follicles were lined by cells, which exhibited nuclear features of PTC-that is, nuclear enlargement, elongation, chromatin clearing, grooves, and inclusions (Fig. 1C, D). The nuclear features of PTC were more evident in the peripheral rim or beneath the capsule of the lesion. In addition, there were areas of hyalinization consisting of bands of eosinophilic fibrous tissue, which were multifocally present throughout the lesions. It is interesting that some of this fibrous tissue seemed to envelop the individual follicles. Psammoma bodies were present in one case. Invasion into the tumor capsule was present in one case; invasion into the capsular vessels was present in three cases. The surrounding thyroid parenchyma showed multiple foci of papillary microcarcinoma follicular variant in one case, and the remaining four cases showed nodular goiter and foci of nonspecific thyroiditis.

As mentioned, in all cases, the bone metastases showed a microfollicular and a solid and follicular growth pattern in one case (Fig. 2A). It is interesting that in none of the bony metastases were nuclear features of papillary carcinoma seen; all showed follicular and solid areas with round, dark nuclei. All of the available lesions showed positivity with immunostains for thyroglobulin (Fig. 2B).

\section{DISCUSSION}

Until 30 years ago, any thyroid cancer exhibiting more than $50 \%$ follicular growth pattern was classified as follicular carcinoma without regard to the nuclear cytology (13). At present, these follicular tumors are classified on the presence or absence of nuclear features of PTC, regardless of percentage follicular pattern (4). Hence, neoplasms that show invasion but lack specific nuclear changes are follicular carcinomas, and tumors with nuclear features are categorized as follicular variant of PTC (1-5). The distinction between these two tumors has important clinical implications (1-3). Follicular carcinomas are angioinvasive and unicentric and metastasize hematogenously (14-16). FVPTC, whether invasive or not, behaves like usual PTC, with multiple tumor foci in the gland in many cases, lymphatic invasion and nodal metastases, and rare hematogenous spread $(1-3,7-9,12,15-$ 20). Survival rates for FVPTC (at least in the shortterm follow-up data available) seem to be similar to usual PTC $(15,16)$.

Some authors have described FVPTC that diffusely involves the gland, the "diffuse follicular variant" (17) or "aggressive follicular variant" (18) that has behaved clinically as follicular carcinoma with 


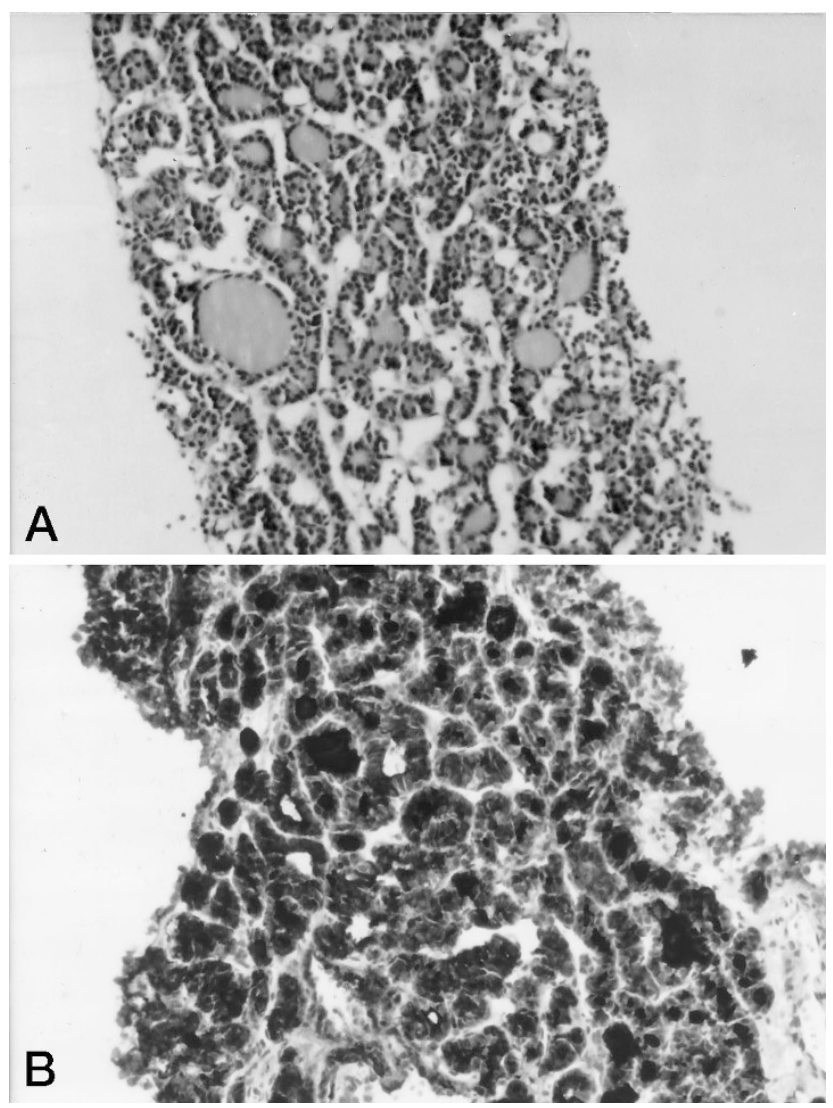

FIGURE 2. Bone core biopsy showing metastatic follicular patterned tumor (A). Staining positive with immunostains for thyroglobulin (B).

distant hematogenous spread to lungs and bone. These tumors seem locally aggressive in the gland with multifocal involvement of both lobes and/or extrathyroidal spread $(17,18)$.

We describe five cases of grossly and microscopically encapsulated follicular patterned neoplasms with nuclei of PTC, hence FVPTC, that did not spread to regional nodes but metastasized to bone. In four of the five cases, the tumors were unifocal. In none did nonosseous sites show metastases at the time of the diagnosis of malignancy.

Despite several reports highlighting the key features of FVPTC, this tumor can be misdiagnosed as follicular carcinoma or follicular adenoma (1-3, 5, 11). The latter usually occurs in cases that are completely encapsulated and show minimal or absent invasive characteristics $(2,3,5)$. The other contributing factor that leads to misdiagnosis of FVPTC as a follicular adenoma is a subset of FVPTC, which shows scattered, multifocal rather than diffuse areas of tumor recognizable as PTC (2, 7, 21-23). Some authors have suggested that an encapsulated follicular lesion with some areas that show nuclear features of PTC and fail to show any invasion should be considered benign (5) because these tumors behave in an indolent fashion.

We report five cases of FVPTC that showed distant bone metastases. All patients were women and
40 years or older. In three cases, the initial thyroid tumor was diagnosed as follicular adenoma. The remaining two cases presented with bone lesions that were found to be metastases from a thyroid primary on the basis of follicular growth pattern and positive thyroglobulin immunostains. All thyroid tumors were completely encapsulated and showed a follicular growth pattern, but multifocally throughout the lesion, the cells exhibited nuclear features of PTC. All cases that initially were diagnosed as follicular adenoma showed one to two foci of vascular invasion on retrospective review. In these cases, the bone metastases were discovered 7 to 17 years after the resection of thyroid lesion. The remaining two cases that presented with bone metastases failed to show any vascular invasion. At the initial surgery, all five patients showed no evidence of lymph node metastases.

The bone metastases from all cases showed solid and follicular growth patterns and were positive for thyroglobulin stains. However, all cases failed to reveal the classic nuclear morphology of PTC. We believe that this is synonymous to the lymph node metastases from papillary carcinoma, in which some cases show only follicular growth pattern and positive thyroglobulin stain and lack nuclear features of PTC (1).

We present these cases to illustrate that (1) encapsulated FVPTC with multifocal rather than diffuse presence of nuclear features of PTC is a lesion that can be mistaken for follicular adenoma; (2) a small subset of encapsulated FVPTC can behave in a more clinically aggressive fashion than classic PTC; (3) these tumors are more common in patients 40 years or older; and (4) although, most FVPTC are clinically similar to classic PTC, a subset of these may clinically behave as hybrids between follicular carcinoma and PTC.

After presenting our suggestions, the question that arises is, "How should one report an encapsulated follicular derived lesion that shows multifocal rather than diffuse distribution of nuclear features of papillary carcinoma?" Should the entire nodule be reported as PTC or all foci of PTC be measured in a lesion and reported separately in a lesion? Vickery (5) reported a few cases of encapsulated papillary carcinoma that showed adenomatous areas intermixed with foci of papillary carcinoma. He suggested that this transition might represent malignant transformation of benign follicular epithelium. Albores-Saavedra et al. (22) reported 17 cases of encapsulated papillary carcinoma that they termed macrofollicular variant. These cases on low power resembled hyperplastic nodules. All of these tumors had randomly distributed foci of FVPTC, which were intermixed with benign-seeming follicles. Two of the cases in original series showed 
lymph node metastasis and maintained the macrofollicular architecture. In a previous immunohistochemical study from our institution based on the expression of cytokeratins in FVPTC, we found that CK19 stained the areas diagnostic of FVPTC as well as areas resembling benign follicles appearing within the lesions. This staining pattern suggested that areas in FVPTC that fail to show the papillary cancer nuclei share a similar phenotype to diagnostic areas of PTC (23).

On the basis of the previously mentioned findings, we believe that encapsulated follicular derived lesions that show multifocal nuclear features of PTC are FVPTC; the whole nodule should be classified as carcinoma for staging and treatment purposes. Although it seems, on the basis of the relatively little and mostly short-term follow-up data, that a majority of these tumors will behave in an indolent fashion, some can metastasize to distant sites.

\section{REFERENCES}

1. LiVolsi VA. Surgical pathology of the thyroid. Philadelphia: W.B. Saunders; 1990.

2. Rosai J, Carcangui ML, DeLellis RA. Tumors of the thyroid gland (Atlas of Tumor Pathology, 3rd series, fascicle 5). Washington, DC: Armed Forces Institute of Pathology; 1993.

3. Hedinger C, Williams ED, Sobin LH. Histological typing of thyroid tumors. WHO international histological classification of tumors. 4th ed. Berlin: Springer-Verlag; 1988.

4. Hawk WA, Hazard JB. The many appearances of papillary carcinoma of the thyroid. Cleve Clin Q 1976;43:207-16.

5. Vickery AL. Thyroid papillary carcinoma: pathological and philosophical controversies. Am J Surg Pathol 1983;7:797803.

6. LiVolsi VA. Papillary neoplasm of the thyroid: pathologic and prognostic features. Am J Clin Pathol 1992;97:426-34.

7. LiVolsi VA. Well differentiated thyroid carcinoma. Clin Oncol 1996;8:281-8.

8. Evans HL. Follicular neoplasms of the thyroid. A study of 44 cases followed for a minimum of 10 years, with emphasis on differential diagnosis. Cancer 1984;54:535-40.

9. Yasumoto K, Miyagi C, Nakashima T, Baba H, Katsuta Y. Papillary and follicular thyroid carcinoma: the treatment results of 357 patients at the National Kyushu Cancer Center of Japan. J Laryngol Otol 1996;110:657-62.

10. Lindsay S. Carcinoma of the thyroid gland: a clinical and pathologic study of 293 patients at the University of California Hospital. Springfield, IL: Charles C Thomas; 1960.

11. Chen KTK, Rosai J. Follicular variant of thyroid papillary carcinoma: a clinicopathologic study of six cases. Am J Surg Pathol 1977;1:123-30.

12. Rosai J, Zampi G, Carcangui ML. Papillary carcinoma of the thyroid. A discussion of its several morphologic expressions, with particular emphasis on the follicular variant. Am J Surg Pathol 1983;8:809-17.

13. Meissner W, Warren S. Tumors of the thyroid gland (Atlas of tumor pathology, series 2, fascicle 3). Washington, DC: Armed Forces Institute of Pathology; 1969.

14. Shaha AR, Loree TR, Shah JP. Prognostic factors and risk group analysis in follicular carcinoma of the thyroid. Surgery 1995;118:1131-8.

15. Mazzaferri EL, Young RL, Oertel JE, Kemmerer WT, Page CP. Papillary thyroid carcinoma: the impact of therapy in 576 patients. Medicine 1977;56:171-96.

16. Mazzaferri EL, Jhiang S. Long-term impact of initial surgical and medical therapy on papillary and follicular thyroid cancer. Am J Med 1994;19:418-28.

17. Sobrinho-Simoes M, Spars J, Carneiro F, Limbert E. Diffuse follicular variant of papillary carcinoma of the thyroid: report of eight cases of a distinct aggressive type of thyroid tumor. Surg Pathol 1990;3:189-203.

18. Guo X, Kleiner D, Fischette M, Merino MJ. Aggressive follicular variant of papillary carcinoma [Abstract]. Mod Pathol 1999;12:67A.

19. Mizukami Y, Noguchi M, Michigishi T, Nonomura A, Hashimoto T, Otakes S, et al. Papillary thyroid carcinoma in Kanazawa, Japan: prognostic significance of histological subtypes. Histopathology 1992;20:243-50.

20. Tielens ET, Sherman SI, Hurban RH, Ladenson PW. Follicular variant of papillary thyroid carcinoma. A clinicopathologic study. Cancer 1994;73:424-31.

21. Baloch ZW, Gupta PK, Yu GH, Sack MJ, LiVolsi VA. Follicular variant of papillary carcinoma. Cytologic and histologic correlation. Am J Clin Pathol 1999;111:216-22.

22. Albores-Saavedra J, Gould E, Vardaman C, Vuitch F. The macrofollicular variant of papillary thyroid carcinoma: a study of 17 cases. Hum Pathol 1991;22:1195-205.

23. Baloch ZW, Abraham S, Roberts S, LiVolsi VA. Differential expression of cytokeratins in follicular variant of papillary carcinoma: an immunohistochemical study and its diagnostic utility. Hum Pathol 1999;30:1166-71. 